\title{
Average Error Rate Expressions for DF relay Networks with CE Error for PSA-CE schemes Over Rayleigh Fading Channels
}

\author{
Chungha Bong, Wonehee Jo and Kyunbyoung Ko* \\ Korea National University of Transportation \\ kbko@ut.ac.kr
}

\begin{abstract}
This paper provides a framework for evaluating the error rate performance of decode and forward (DF) relay cooperative transmission in the presence of channel estimation (CE) error caused by pilot symbol assisted-channel estimation (PSA-CE) schemes over Rayleigh fading channels. Average bit error rate (BER) and average symbol error rate (SER) are expressed as the well-known closed-forms by using error-events at relay nodes and moment generating function (MGF) of the received signal-to-noise ratio (SNR), which quantifies the SNR penalty arising from CE errors. Moreover, the effects of erroneous detection and transmission at relay nodes are verified in terms of both the combined SNR and the average error rate, and cooperative diversity is observed from closed-form error rate expressions. Simulation results are finally presented to validate derived analytical performance ma-trices.
\end{abstract}

Keywords: DF relay, CH, PSA-CE, Rayleigh fading channels, BER, SER, MGF, SNR

\section{Introduction}

Cooperative diversity networks have recently been widely discussed for wireless systems $[1,2]$. Effects of cooperative diversity in radio communication have been studied [3, 4]. In decode-and-forward (DF) relay networks, the relay detects the received signal and then retransmits a regenerated signal $[1,2]$. At the destination, the receiver can employ a variety of diversity combining techniques to benefit from the multiple signal replicas available from the relays and the source. Use of DF schemes presumes the incorporation of a cyclic redundancy check (CRC) codes from a higher layer (e.g., data link layer) in order to detect errors. The advantages of general cooperative diversity schemes come at the expense of the spectral efficiency since the source and all the relays must transmit on orthogonal channels (i.e., different time slots or frequency bands) in order to avoid interfering with each other as well [2]. Recent researches are carried out for relay-selection schemes in which only two channels are need (one for the direct link and the other one for best relay link) [5-8]. However, they need to additional process or feedback information for channel states.

The authors in [9] have shown exact and closed-form expressions for bit error rate (BER) of DF relaying with multiple relays in dissimilar Rayleigh fading channels. In [10], the authors investigated the error performance of DF relaying over Nakagami-m fading channels. But, the analytical approaches in [9] and [10] can be applied to only DF schemes (excluding fixed-DF (FDF)). In [9], the correctly decoded set at relay nodes is based so that derived results have not been expressed as the well-known tractable form. Recently, the general approach covering both FDF and DF relaying has been addressed in [11]. The authors in [11] have expressed the exact BERs as well known tractable forms, which can be explain how an

* Corresponding Author 
erroneous detection at each relay affects both the received signal-to-noise ratio (SNR) and the average BER. In [12], the authors provided a framework for evaluating the BER performance of DF relay-assisted cooperative transmission in the presence of imperfect channel estimation. Nevertheless, a framework in [12] cannot be applied to pilot symbol assisted-channel estimation (PSA-CE) schemes which can be practical ones. Not that the error rate performance in [12] gives error-floor even at high SNR region. It is impractical results for (PSA-CE) schemes.

So far as we know, the general approach based on PSA-CE schemes for DF relaying has not been addressed in the literature yet. Furthermore, no one has expressed average error rate expressions with $\mathrm{CE}$ generated from PSA-CH schemes, which can be explain how an erroneous detection at each relay and CE error affect both the received SNR and the average error rates. The remainder of the paper is organized as follows: Section II describes the system model for DF relay networks with PSA-CE schemes. In Section III, the average error rate expressions are provided. The numerical results are presented in Section IV and concluding remarks are given in Section V.

\section{DF Relay Networks}

Figure 1. shows the block diagram of DF relay systems with a Source(S), a destination (D), and a relay $(\mathrm{R})$. The number of relays is $L$. In this paper, it is assumed that $\mathrm{S}$ and $\mathrm{R}$ relays transmit over orthogonal frequency bands. At first, let us describe the quasi-static Rayleigh fading channel model to derive the analytical approach for PSA-CE scheme.

\subsection{DF Relay System Model}

Let $h_{0}, h_{L+r}$, and $h_{r}$ with $r \in\{1,2, \cdots, L\}$ be the channel gains of S-D, S-R, and R-D link channels, respectively. In this paper, wireless channels between any pair of nodes are assumed quasi-static independent and non-identical distributed (INID) Rayleigh fading [1315]. In means that channel coefficient can be assumed to be a constant during the several symbol times (i.e., during a frame transmission). From here, let us define $N_{P}$ and $N_{D}$ as the number of pilots and the number of modulated data symbols within a frame so that the length of frame is $N_{F}\left(=N_{P}+N_{D}\right)$.

The received signals for S-D, S-R, and R-D links are presented, respectively, as

$$
\begin{array}{ll}
y_{0}[t] & =h_{0} s[t]+n_{0}[t] \\
y_{L+r}[t] & =h_{L+r} s[t]+n_{L+r}[t] \\
y_{r}[t] & =h_{r} \hat{s}_{r}[t]+n_{r}[t]
\end{array}
$$

where $t \in\left\{1,2, \cdots, N_{F}\right\}$ is the time index within a given frame, and each channel is corrupted by complex additive white Gaussian noise (AWGN) term of $n_{r}[t]$. Without loss of generality, we can assume that $\left\{n_{r}[t]\right\}$ are mutually independent for different $r$ and $t$ with $E\left[\left|n_{r}[t]\right|\right]=0$ and $E\left[\left|n_{r}[t]\right|^{2}\right]=\sigma^{2}$. The operator $E[\cdot]$ represents statistical expectation. For simplicity, we consider in this paper the first frame transmission. Then, $\left.s[t]\right|_{1 \leq t \leq N_{P}}$ are the pilot symbols known to all relay nodes and the destination and $\left.s[t]\right|_{N_{P}<t \leq N_{F}}$ are M-ary phase 
shift keying (MPSK) data symbols. Also, $\{s[t]\}$ are mutually independent for different ${ }^{t}$ with $E[s[t]]=0$ and $E\left[|s[t]|^{2}\right]=1$. In DF relay systems, the $r$ th relay is only to transmit the regenerated symbol of $\hat{s}_{r}[t]$ when messages are correctly decoded. It means that the channel condition of the $r$ th S-R link is sufficiently good to allow for successful decoding [5]. In addition, it is assumed that the relay node is capable of perfect forwarding the original message if we can detect an error for each bit. Note that $\left.\hat{s}_{r}[t]\right|_{1 \leq t \leq N_{P}}=s[t]$ is pilot symbols to estimate R-D link channel.

\subsection{PSA-CH Schemes}

For PSA-CE schemes, the channel coefficients can be estimated over quasi-static fading channels as

$$
\hat{h}_{r}=\frac{1}{N_{p}} \sum_{t=1}^{N_{P}} s^{*}[t] y_{r}[t]=h_{r}+e_{r}
$$

where the CE error is $e_{r}=\frac{1}{N_{p}} \sum_{t=1}^{N_{P}} S^{*}[t] n_{r}[t]$ with $E=\left[\left|e_{r}\right|^{2}\right]=\sigma^{2} / N_{P}$. Note that $\left.S[t]\right|_{N_{P}<t \leq N_{F} \text { with }}|S[t]|^{2}=1$ are pilot symbols known to the destination and all relays. By using the known pilot symbols and the estimated channel coefficient of (2), the noise variance can be estimated as

$$
\hat{\sigma}_{r}^{2}=\frac{1}{N_{P}} \sum_{p=1}^{N_{P}}\left|y_{r}[t]-\hat{h}_{r} s[t]\right|^{2} .
$$

Note that for the large $N_{P}$, the estimated noise variance of (3) can be approximated as

$$
\hat{\sigma}_{r}^{2} \approx \tilde{\sigma}_{r}^{2}=E\left[\left|y_{r}[t]-\hat{h}_{r} s[t]\right|^{2}\right]=\frac{N_{P}-1}{N_{P}} \sigma^{2} .
$$

\subsection{Performance Analysis for S-R link with CE Errors}

Using $\hat{h}_{L+r}$ and $\hat{\sigma}_{L+r}^{2}$ for the $r$ th S-R link, the decision variable for data symbols can be written as

$$
\left.Z_{r}[t]\right|_{N_{P}<t \leq N_{F}}=\left(\hat{h}_{L+r}\right)^{*} y_{L+r}[t] / \hat{\sigma}_{L+r}^{2}
$$

and from $\hat{h}_{L+r}=h_{L+r}+e_{L+r}$ in (2), the received instantaneous SNR is expressed as

$$
\gamma_{L+r}=\frac{\left|\hat{h}_{L+r}\right|^{2}}{\sigma^{2}\left(N_{P}+1\right) / N_{P}} .
$$


Then, the probability density function (PDF) of $\gamma_{L+r}$ can be presented for the Rayleigh fading channel as

$$
f_{\gamma_{L+r}}(x)=\frac{1}{\bar{\gamma}_{L+r}} \exp \left(-\frac{x}{\bar{\gamma}_{L+r}}\right)
$$

where $\gamma_{L+r}$ is the average SNR defined as

$$
\bar{\gamma}_{L+r}=E\left[\gamma_{L+r}\right]=\frac{E\left[\left|\hat{h}_{L+r}\right|^{2}\right]}{\sigma^{2}\left(N_{P}+1\right) / N_{P}}
$$

with $E\left[\left|\hat{h}_{L+r}\right|^{2}\right]=E\left[\left|h_{L+r}\right|^{2}\right]+\sigma^{2} / N_{P}$. Furthermore, above derivation can be also applied to S-D and each R-D links so that by replacing $L+r$ with $r$, we can obtain $\gamma_{r}, \bar{\gamma}_{r}$, and $f_{\gamma_{r}}(x)$ with $r \in\{0,1, \cdots, L\}$. Consequently, the average BER of the $r$ th S-R link can be expressed for BPSK as

$$
P_{b}\left(\bar{\gamma}_{L+r}\right)=\frac{1}{2}\left[1-\sqrt{\frac{\bar{\gamma}_{L+r}}{1+\bar{\gamma}_{L+r}}}\right]
$$

and the average SER of the $r$ th S-R link can be written for MPSK as

$$
P_{S}\left(\bar{\gamma}_{L+r}\right)=\frac{1}{\pi} \int_{0}^{(M-1) \pi / M} M_{L+r}(s) d \theta
$$

with $s=\frac{g_{P S K}}{\sin ^{2} \theta}, g_{P S K}=\sin ^{2}(\pi / M)$, and $M_{L+r}(s)=\left(1+s \bar{\gamma}_{L+r}\right)^{-1}$ which is the MGF of random variable $\gamma_{L+r}[13][14]$.

Note that for $N_{P} \rightarrow \infty$,

$$
\begin{aligned}
& \lim _{N_{P} \rightarrow \infty} E\left[\left|e_{r}\right|^{2}\right]=0 \\
& \lim _{N_{P} \rightarrow \infty} \bar{\gamma}_{L+r}=\frac{E\left[\left|h_{L+r}\right|^{2}\right]}{\sigma^{2}} .
\end{aligned}
$$

From above equation, we can find the fact that when the number of pilots increase, the CE error is decrease (i.e., the estimated one approaches ideal channel gain) and the average SNR merges to the case of ideal channel estimation.

\section{Performance Analysis of DF Relay Systems with CE Errors from PSA- CE Schemes}

In DF relay systems, the $r$ th relay participates in transmitting the regenerated symbol of $\hat{s}_{r}[t]$ only when messages are correctly decoded. Then, $\hat{s}_{r}[t]$ can be two values, which are 
$\hat{s}_{r}[t]=0$ with the probability of $P_{b}\left(\bar{\gamma}_{L+r}\right)$ or $P_{S}\left(\bar{\gamma}_{L+r}\right)$ and $\hat{s}_{r}[t]=s[t]$ with the probability of $1-P_{b}\left(\bar{\gamma}_{L+r}\right)$ or $1-P_{S}\left(\bar{\gamma}_{L+r}\right)$.

\subsection{Error-Event of Relay Nodes}

In order to generally derive the analytical method based on error-events at relays, let us define the $p_{\text {th error-event vector }} E^{p}$ as $[10,11]$

$$
E^{p}=\left[e_{1}^{p} \cdots e_{r}^{p} \cdots e_{R}^{p}\right]
$$

with $p \in\left\{1,2, \cdots, 2^{R}\right\}$ and the total number of error-events is $2^{R}$. Generally, we can define that $E^{1}$ is all-zero vector, $E^{2^{R}}$ is all-one vector, and so on. Note that for the $p_{\text {th error-event, }}$ $e_{r}^{p}=0$ means the correct detection at the $r$ th relay and $\left.\hat{s}_{r}[t]\right|_{N_{P}<t \leq N_{F}}=s[t]$ with the probability of $1-P_{b}\left(\bar{\gamma}_{L+r}\right)$ (or $1-P_{S}\left(\bar{\gamma}_{L+r}\right)$ ). Also, $e_{r}^{p=1}$ leads to $\left.\hat{s}_{r}[t]\right|_{N_{P}<t \leq N_{F}}=0$ with the probability of $P_{b}\left(\bar{\gamma}_{L+r}\right)$ (or $P_{S}\left(\bar{\gamma}_{L+r}\right)$ ).

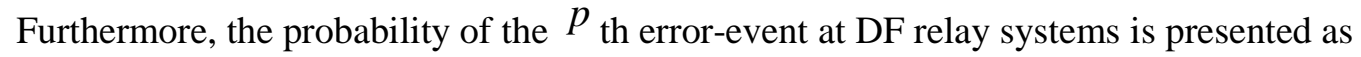

$$
\begin{aligned}
& \operatorname{Pr}_{b}^{p}=\prod_{r=1}^{R}\left[1-P_{b}\left(\bar{\gamma}_{L+r}\right)\right]^{e_{r}^{p}}\left[P_{b}\left(\bar{\gamma}_{L+r}\right)\right]^{e_{r}^{p}} \\
& \operatorname{Pr}_{S}^{p}=\prod_{r=1}^{R}\left[1-P_{S}\left(\bar{\gamma}_{L+r}\right)\right]^{e_{r}^{p}}\left[P_{S}\left(\bar{\gamma}_{L+r}\right)\right]^{e_{r}^{p}}
\end{aligned}
$$

$$
\text { with } \overline{e_{r}^{p}}=\left(e_{r}^{p}+1\right) \bmod 2 \text { [11]. }
$$

\subsection{Average BER Expression}

At the destination node, a maximal ratio combing (MRC) scheme can be applied in order to combine signals from S-D and R-D links. For MRC, the noise variance normalization process is necessary in order to fully obtain the diversity gain so that we need not only estimated channel coefficients but also estimated noise variances. Using $\hat{h}_{r}$ and $\hat{\sigma}_{r}^{2}$, the decision variable for the $p$ th error-event can be obtained as

$$
\left.z_{\text {tot }}^{p}[t]\right|_{N_{P}<t \leq N_{F}}=\sum_{r=0}^{L}\left(\hat{h}_{r}\right)^{*} \overline{e_{r}^{p}} y_{r}[t] / \hat{\sigma}_{r}^{2} .
$$

Note that in order to derive the combined instantaneous SNR, we need the approximation for (14) with $\hat{\sigma}_{r}^{2}$ of (3) as the form related with $\tilde{\sigma}_{r}^{2}$ of (4). It leads to

$$
z_{\text {tot }}^{p}[t] \approx \sum_{r=0}^{L}\left(\hat{h}_{r}\right)^{*} \overline{e_{r}^{p}} y_{r}[t] / \tilde{\sigma}_{r}^{2}
$$


and from (15), the instantaneous SNR can be modified as $\gamma_{\text {tot }}^{p}=\gamma_{0}+\sum_{r=1}^{R} \overline{e_{r}^{p}} \gamma_{r}$. It means that when there is a detection-error at the $r$ th relay node for the $p_{\text {th event vector (i.e., }}$ $e_{r}^{p}=1$ ), no-transmission give $\overline{e_{r}^{p}} \gamma_{r}=0$. Therefore, in DF schemes, $\overline{e_{r}^{p}}$ can be regarded as

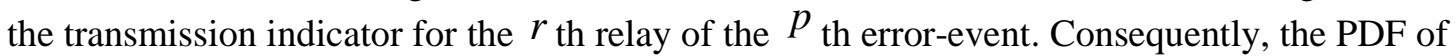
$\gamma_{\text {tot }}^{p}$ can be presented as

$$
f_{\gamma_{\text {tot }}^{p}}(x)=\sum_{r=0}^{R} \frac{\pi_{r}^{p}}{\bar{\gamma}_{r}} \exp \left(\frac{-\overline{e_{r}^{p}} x}{\bar{\gamma}_{r}}\right)
$$

with $\pi_{r}^{p}=\prod_{i=0, i \neq r}^{R} \frac{\overline{e_{r}^{p}} \bar{\gamma}_{r}}{\overline{e_{r}^{p}} \bar{\gamma}_{r}-\overline{e_{i}^{p}} \bar{\gamma}_{i}}$ [11]. Note that for S-D link terms in (16), we can define $\overline{e_{0}^{p}}=1$ so as to $\overline{e_{0}^{p}} \bar{\gamma}_{0}=\bar{\gamma}_{0}$.

Consequently, the conditional BER for BPSK can be presented as

$$
P_{b}^{p}\left(\gamma_{\text {tot }}^{p}\right)=Q\left(\sqrt{2 \gamma_{\text {tot }}^{p}}\right)
$$

with $Q(\sqrt{2 x})=1 / \sqrt{2 \pi} \int_{\sqrt{2 x}}^{\infty} \exp \left(-t^{2} / 2\right) d t$ and the conditional BER of (17) is averaged over the PDF given in (16), the result is

$$
P_{b}^{p}=\frac{1}{2} \sum_{r=0}^{R} \pi_{r, D F}^{p}\left[1-\sqrt{\frac{\overline{e_{r}^{p}} \bar{\gamma}_{r}}{1+\overline{e_{r}^{p}} \bar{\gamma}_{r}}}\right]
$$

Then, taking into account for all the possible error-events, the average BER is presented as

$$
P_{b}=\sum_{p=1}^{2^{R}} \operatorname{Pr}^{p} P_{b}^{p}
$$

Note that for the special case, when all relays undergo incorrect-detection, we can have $\gamma_{\text {tot }}^{2^{R}}=\gamma_{0}$ and $P_{b}^{2^{R}}=P_{b}\left(\bar{\gamma}_{0}\right)$.

\subsection{Average SER Expression}

By utilizing the moment generating function (MGF), the average SER can be derived. At first, the conditional SER for MPSK of the $p$ th error-event is written as

$$
P_{S}^{p}\left(\gamma_{\text {tot }}^{p}\right)=\frac{1}{\pi} \int_{0}^{(M-1) \pi / M} \exp \left(-\frac{g_{P S K} \gamma_{t o t}^{p}}{\sin ^{2} \theta}\right) d \theta
$$

and the average SER can be presented as

$$
P_{S}^{p}=\frac{1}{\pi} \int_{0}^{(M-1) \pi / M} \prod_{r=0}^{R} M_{r}(s) d \theta
$$


with and

$$
M_{r}(s)=\frac{1}{1+s \overline{e_{r}^{p}} \bar{\gamma}_{r}}
$$

which is the MGF of random variable $\overline{e_{r}^{p}} \gamma_{r}$. Finally, considering all the possible errorevents, the average SER is presented as

$$
P_{S}=\sum_{p=1}^{2^{R}} \operatorname{Pr}^{p} P_{S}^{p} .
$$

\section{Numerical and Simulation Result}

In this section, we show numerical results of average error rates and verify their accuracy by comparing simulation results. We assumed for the channel conditions that $E\left[\left|h_{L+r}\right|^{2}\right]=E\left[\left|h_{0}\right|^{2}\right] \quad, \quad E\left[\left|h_{r}\right|^{2}\right]=E\left[\left|h_{0}\right|^{2}\right] / R \quad$ for $\quad r \in\{1,2, \cdots, L\} \quad, \quad$ and $S N R=E\left[\left|h_{0}\right|^{2}\right] / \sigma^{2}$.

Figure 2 shows the average error rates versus SNR for the DF relay systems with $M=2$, $L=4$, and $N_{P} \in\{0,2,4,8\}$. Note that $N_{P}=0$ means the ideal channel estimation in witch analytical results exactly match with simulated ones. From this figure, we can find that for the case of $N_{P} \neq 0$, there are mismatches between analytical results and simulated ones. Those mismatches can be caused by the approximation related with the derivation of received SNR (i.e., eq. (15)). Furthermore, those mismatches decrease in proportion to $N_{P}$. Fig. 3 shows the average error rate comparison versus the source-to-destination link's SNR with respect to different $L \in\{1,2,4\}$. It can be noticed form Figure 3 with $N_{P}=8$ that the derived analytical results can be well-matched with simulated ones. Moreover, we can find that the diversity order increases linearly with the number of relay $L$. Consequently, it is confirmed that the derived analytical approach can be used as a general tool to verify effects of PSACHE on the average error rate and cooperative diversity gain over quasi-static Rayleigh fading channel.

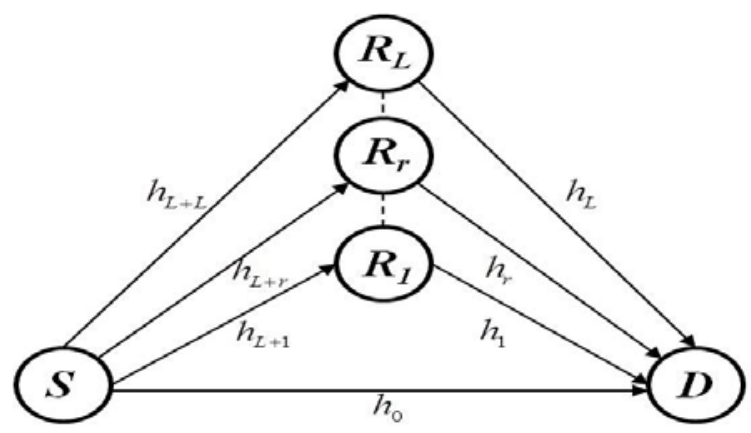

Figure 1. Block Diagram of DF Relay Systems(source(S), Destination(D), relay( $R)$ ) 


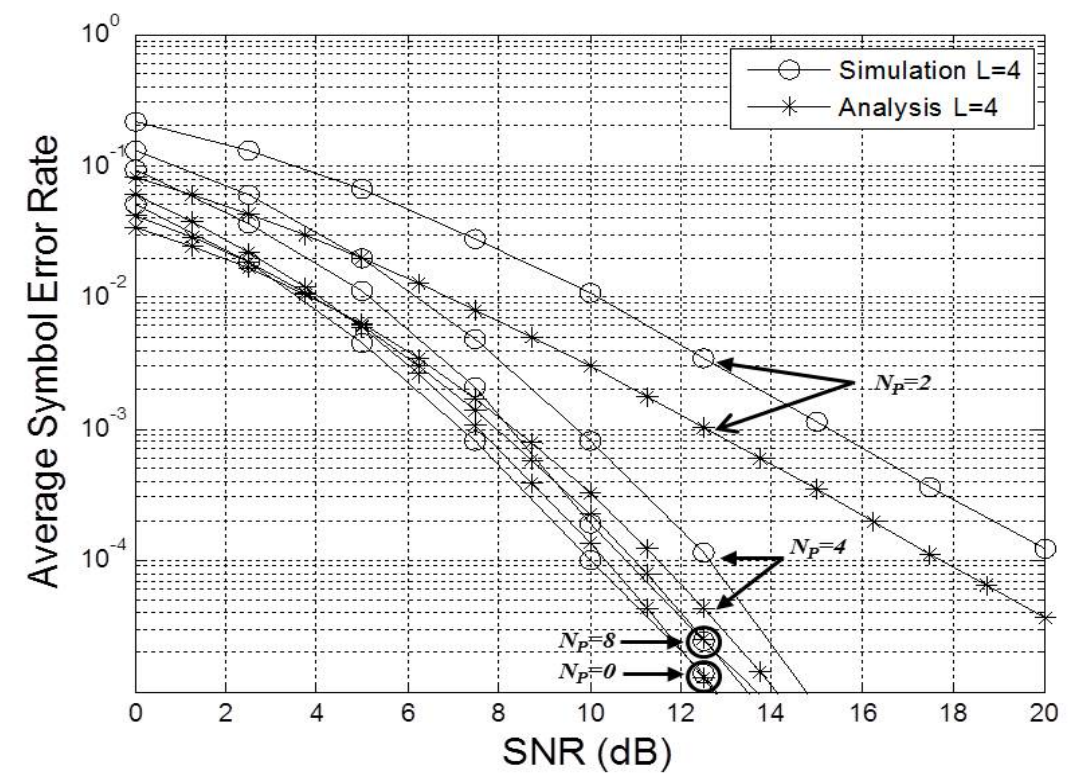

Figure 2. Averaged BER versus SNR for DF Relay Systems with Respect to Different Number of Pilot Symbol Nodes

$$
\left(S N R=E\left[\left|h_{0}\right|^{2}\right] / \sigma^{2}, M=2, L=4, N_{P}=0,2,4,8\right)
$$

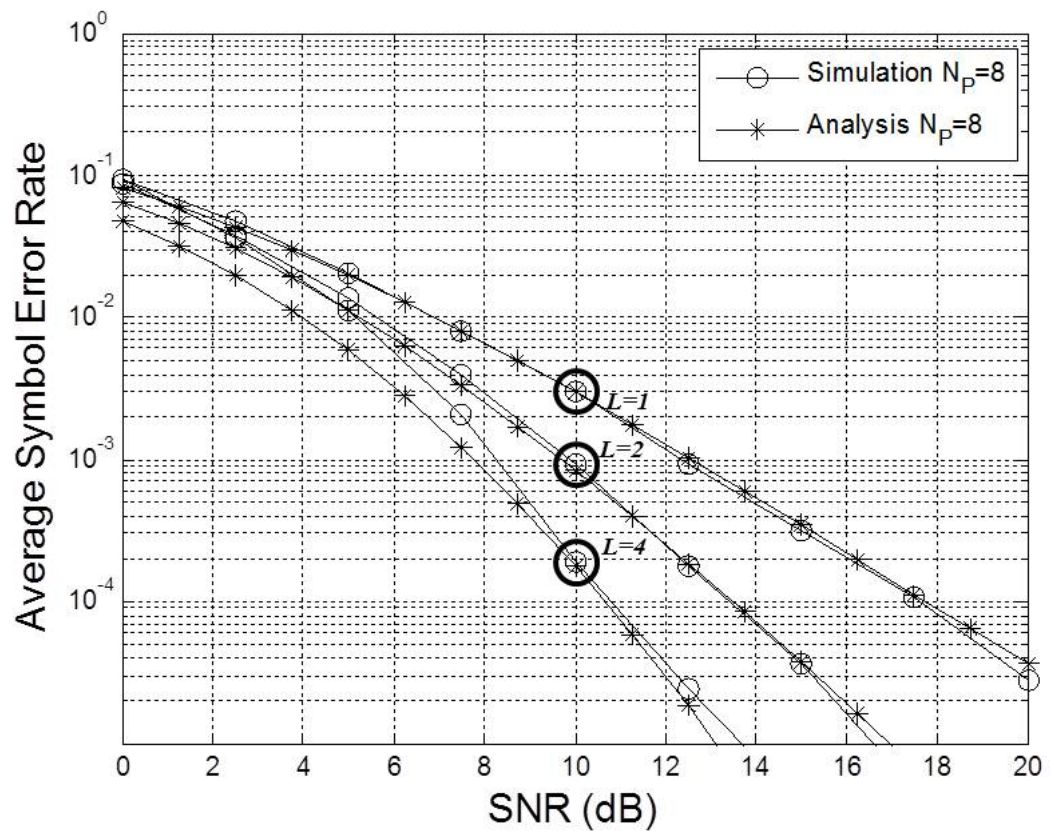

Figure 3. Averaged BER versus SNR for DF Relay Systems with Respect to Different Number of Relay Nodes ( $S N R=E\left[\left|h_{0}\right|^{2}\right] / \sigma^{2}, M=2, L=1,2,4, N_{P}=8$ ) 


\section{Conclusions}

In this paper, we have developed an analytical method for average error rate expression related with PSA-CHE based on error-events at relay nodes of DF relay systems over quasistatic Rayleigh fading channels. The well-known form error rate expressions were evaluated as both average BER and SER. For the case of large number of pilots, we were able to verify that our approach well matches with the simulation results. Under the general cooperative diversity scheme, we expect our theoretical results to be useful for further analysis and in the evaluation of practical implementations

\section{Acknowledgements}

This research was supported by Basic Science Research Program through the National Research Foundation of Korea(NRF) funded by the Ministry of Education, Science and Technology(2012-0003625, 2013-04360001).

\section{References}

[1] M. O. Hasna and M.-S. Alouini, IEEE Trans. Wireless Commun., vol. 2, no. 6, (2003) November, pp. 11261131.

[2] J. N. Laneman, D. N. C. Tse and G. W. Wornell, IEEE Trans. Inf. Theory, vol. 50, no. 12, (2004) December, pp. 3062-3080.

[3] A. Nandi and S. Kundu, International Journal of Future Generation Communication and Networking, vol. 4, no. 2, (2011) June, pp. 91-104.

[4] C. Kumar De and S. Kundu, International Journal of Future Generation Communication and Networking, vol. 5, no. 1, (2012) March, pp. 29-42.

[5] A. Bletsas, A. Khisti, D. P. Reed and A. Lippman, IEEE J. of Selected Areas in Commun., vol. 24, no. 3, (2006) March, pp. 659-672.

[6] K. Ko and C. Woo, International Journal of Communication Systems, vol. 25, no. 11, (2012) November, pp. 1496-1504.

[7] S. Nam, K. Ko and D. Hong, “IEICE Trans. on Commun., E95-B(5), (2012) May, pp. 1852-1855.

[8] K. Ko and C. Woo, "Wireless Personal Communications, vol. 68, no. 3, (2013) February, pp. 609-617.

[9] I.-H. Lee and D. Kim, IEEE Commun. Lett., vol. 11, no. 1, (2007) January, pp. 52-54.

[10] Y. Lee, M.-H. Tsai and S.-I. Sou, IEEE Trans. Wireless Commun., vol. 8, no. 6, (2009) June, pp. 2853-2859.

[11] J. Y. Jang and K. B. Ko, "IEICE Trans. on Commun., E94-B( 08), (2011) August, pp. 2419-2422.

[12] S. Han, S. Ahn, E. Oh and D. Hong, IEEE Trans. Veh. Technol., vol. 58, no. 4, (2009) May, pp. 2083-2088.

[13] J. G. Proakis, “Digital Communication”, McGraw Hill, New York, (1995).

[14] M. K. Simon and M.-S. Alouini, "Digital Communication over Fading Channels”, John Wiley \& Sons, New York, (2000).

[15] D. Alam and R. Huque Khan, International Journal of Future Generation Communication and Networking, vol. 6, no. 2, (2013) April, pp. 11-24.

\section{Authors}

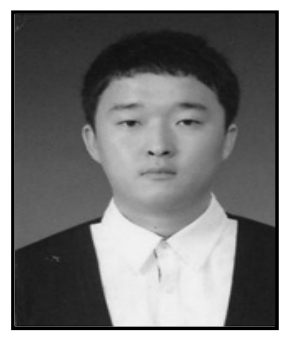

Chungha Bong, received the B.S. and M.S. degrees in the Department of Control and Instrumentation Engineering at the Korea National University of Transportation, Chungju, Korea in 2005 and 2007, respectively. From 2012, he is working toward the Ph.D. degree in the Department of Control and Instrumentation Engineering at the Korea National University of Transportation. His current research interests include the field of wireless communications focusing on multicarrier systems, cooperative relaying, and cognitive radio. 


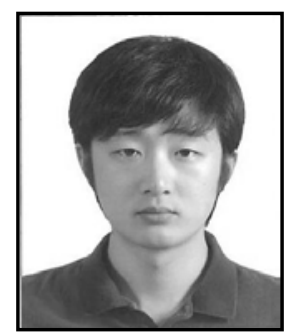

Wonehee Jo, received the B.S. degrees in the Department of Control and Instrumentation Engineering at the Korea National University of Transportation, Chungju, Korea in 2012. Currently, he is working toward the M.S. degree in the Department of Control and Instrumentation Engineering at the Korea National University of Transportation. His current research interests include the field of wireless communications focusing on cooperative relaying and cognitive radio.

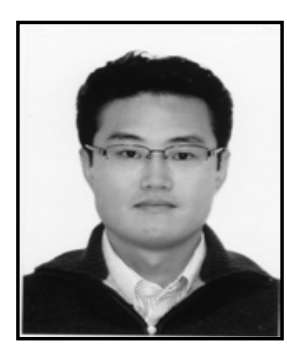

Kyunbyoung Ko, received the B.S., M.S., and Ph.D. degrees in Electrical and Electronic Engineering at Yonsei University, Seoul, Korea in 1997, 1999, and 2004, respectively. From March 2004 to February 2007, he was a senior engineer in Samsung Electronics, Suwon, Korea where he developed Mobile WiMAX systems for broadband wireless services. In March 2007, he joined the Department of Control and Instrumentation Engineering at the Korea National University of Transportation as an associate professor. His current research interests include the field of wireless communications focusing on multicarrier and multi-antenna systems, cooperative relaying, cognitive radio, and intelligent transportation system ( ITS). 